\title{
Identification of dysregulated long non-coding RNAs/microRNAs/ mRNAs in TNM I stage lung adenocarcinoma
}

\author{
Ziqiang Tian ${ }^{1}$, Shiwang Wen ${ }^{1}$, Yuefeng Zhang ${ }^{1}$, Xinqiang Shi ${ }^{1}$, Yonggang Zhu ${ }^{1}$, \\ Yanzhao Xu' ${ }^{1}$, Huilai Lv ${ }^{1}$ and Guiying Wang ${ }^{2}$ \\ ${ }^{1}$ Department of Thoracic Surgery, The Fourth Hospital of Hebei Medical University, Shijiazhuang, China \\ ${ }^{2}$ The Second Department of Surgery, The Fourth Hospital of Hebei Medical University, Shijiazhuang, China \\ Correspondence to: Guiying Wang, email: tizq12@vip.163.com \\ Keywords: TNM I stage, lung adenocarcinoma, long non-coding RNA, microRNA, RNA-sequencing \\ Received: December 29, $2016 \quad$ Accepted: May 06, $2017 \quad$ Published: June 16, 2017 \\ Copyright: Tian et al. This is an open-access article distributed under the terms of the Creative Commons Attribution License 3.0 \\ (CC BY 3.0), which permits unrestricted use, distribution, and reproduction in any medium, provided the original author and source \\ are credited.
}

\section{ABSTRACT}

Lung adenocarcinoma (LUAD) is the primary subtype in lung cancer, which is the leading cause of cancer-related death worldwide. This study aimed to investigate the aberrant expression profiling of long non-coding RNA (IncRNA) in TNM I stage (stage I) LUAD. The IncRNA/mRNA/miRNA expression profiling of stage I LUAD and adjacent non-tumor tissues from 4 patients were measured by RNA-sequencing. Total of 175 differentially expressed IncRNAs (DELs), 1321 differentially expressed mRNAs (DEMs) and 94 differentially expressed microRNAs (DEMIs) were identified in stage I LUAD. DEMI-DEM regulatory network consisted of 544 nodes and 1123 edge; miR-200 family members had high connectivity with DEMs. In DEL-DEM coexpression network, CDKN2B-AS1, FENDRR and LINC00312 had the high connectivity with DEMs, which co-expressed with 105, 63 and 61 DEMs, respectively. DEL-DEMIDEM network depicted the links among DELs, DEMI and DEMs. Identified DEMs were significantly enriched in cell adhesion molecules, focal adhesion and tight junction of Kyoto Encyclopedia of Genes and Genomes (KEGG) pathways; and enriched in cell adhesion, angiogenesis and regulation of cell proliferation of Gene Ontology biological processes. Quantitative real-time polymerase chain reaction results were generally consistent with our bioinformatics analyses. LINC00312 and FENDRR had diagnostic value for LUAD patients in The Cancer Genome Atlas database. Our study might lay the foundation for illumination of pathogenesis of LUAD and identification of potential therapeutic targets and novel diagnosis biomarkers for LUAD patients.

\section{INTRODUCTION}

Lung adenocarcinoma (LUAD), the primary histological subtype of non-small cell lung cancer (NSCLC), is the leading cause of cancer mortality both in female and male around the world [1].

Resection surgery is recognized as the radical treatment for patients with LUAD, However, patients frequently lose opportunity for radical resection on account of most of patients are diagnosed with advanced stage LUAD in hospitalization [2]. In addition, chemotherapy and radiotherapy are the standard first- and second-line treatments for patients with LUAD, but the curative effect remains undesirable [3]. Although epidermal growth factor receptor tyrosine kinase inhibitors (EGFR-TKIs), such as gefitinib and erlotinib, greatly improve the life quality of patients, but EGFRTKIs cannot significantly prolong overall survival and results in inevitable gefitinib-resistance [4]. In spite of the aforementioned advances in clinical therapy, the prognosis 5- year overall survival of patients with LUAD is dissatisfying. Although it is known that early diagnosis and treatment for patients with LUAD will be beneficial to prolong overall survival of patients, but the current 
diagnostic biomarker for early stage LUAD is not feasible in clinical practice on account of unsatisfactory sensitivity and specificity.

Long non-coding RNAs (lncRNAs) are those transcripts with length more than $200 \mathrm{nt}$ and not translated into proteins. Mounting evidences indicate that aberrant expression of lncRNAs is involved in modulating the transcription and translation of protein-coding genes at the transcriptional level, post-transcriptional level and epigenetic level [5]. IncRNAs play essential roles in cell proliferation and metastasis of various cancer types. Ectopic expression of AFAP-AS1 promotes cell proliferation and inhibits cell apoptosis in esophageal squamous cell carcinoma (ESCC), which is significantly correlated with advanced TNM stage and larger tumor size [6]. Over-expression of lncRNA MALAT1 predicts poor recurrence-free survival in tamoxifen treated ER-positive breast cancer patients [7]. In gallbladder, high expression level of MALAT1 is correlated with larger tumor size, lymphatic metastasis and shorter overall survival; silencing of MALAT1 inhibits cell proliferation, cell invasion and increases cell apoptosis [8]. In currently, a few of articles demonstrate the lncRNA expression profiling in LUAD tissues, but the global lncRNA expression profiling in TNM I stage LUAD (stage I LUAD) is not uncovered.

In our work, lncRNA, miRNA and mRNA expression profiling of patients with early stage LUAD were obtained through RNA-sequencing, abnormally expressed lncRNAs, miRNAs and mRNAs were identified, and lncRNA/mRNA co-expression network were constructed. Our study might provide new insights into investigation of tumorigenesis mechanism in LUAD and discover of candidate diagnostic biomarkers and therapeutic targets for LUAD.

\section{RESULTS}

\section{Differentially expressed IncRNAs and mRNAs in TNM I stage LUAD}

Respective 15710 lncRNAs and 22528 mRNAs were mapped with the human reference genome Ensemble GRCh38 v 84. Total of 175 differentially expressed lncRNA (DELs, 63 up- and 112 down-regulated) and 1321 differentially expressed mRNA (DEMs, 587 up- and 734 down-regulated) were identified in TNM I stage LUAD tissues compared to paired non-tumor tissues according to the threshold of $P<0.05$ and $\mid \log _{2}$ Fold change $\mid \geq 1.175$ DELs and 1321 DEMs were widely distributed in all autosomes and chromosomes X (Supplementary Figure 1). The top 15 up- and down-regulated DELs/DEMs were shown in Tables 1 and 2. LOC80078 and LOC101930114 were the most significantly up- and down-regulated DELs; EEF1A2 and ANKRD1 were the most significantly up- and down-regulated DEMs in TNM I stage LUAD tissues compared to paired non-tumor tissues (Tables 1 and 2).

\section{Differentially expressed miRNA (DEMIs) in stage I LUAD}

Total of 94 DEMIs including 87 up- and 7 downregulated DEMIs were identified in TNM I stage LUAD tissues compared with paired non-tumor tissues based on the threshold of FDR $<0.001$ and $\mid \log _{2}$ fold change $\mid \geq 1$. hsamiR-194-5p, hsa-miR-135b-5p and hsa-miR-215-3p were significantly up-regulated in stage I LUAD tissues; hsamiR-486-5p, hsa-miR-338-3p, hsa-miR-7641, hsa-miR138-5p, hsa-miR-451a, hsa-miR-486-3p and hsa-miR$139-3 p$ were significantly down-regulated in early stage LUAD tissues compared with adjacent non-tumor tissues (Supplementary Table 1).

\section{Construction of DEMI-DEM interaction network}

The target-genes of top 15 up- and down-regulated DEMIs in stage I LUAD were predicted though miRWalk database. Those predicted target-genes were overlapped with 1321 DEMs, and DEMI-DEM interaction pairs in a negative regulation manner were our concern due to miRNA commonly negatively regulates the expression of mRNA. DEMI-DEM regulatory network were visualized by Cytoscape software. As Figure 1A shown, up-regulated DEMIs/down-regulated DEMs regulatory network composed of 395 nodes and 942 edges, which was involved in 15 up-regulated DEMIs and 384 DEMs; hsa-miR-182-5p, hsa-miR-200b-3p and hsa-miR-429 had the highest connectivity with down-regulated DEMs, which interacted with 99,83 and 82 DEMs, respectively. As Figure 1B shown, down-regulated DEMIs/up-regulated DEMs regulatory network composed of 139 nodes and 181 edges, which was involved in 7 up-regulated DEMIs and 132 DEMs; hsa-miR-486-3p, hsa-miR-138-5p and hsa-miR-338-3p had the highest connectivity with upregulated DEMs, which interacted with 61, 45 and 41 down-regulated DEMs, respectively.

\section{The nearby DEMs of DELs in reference genome}

The nearby protein-coding genes of 175 DELs with distance $<100 \mathrm{~kb}$ were identified in GRCH38 reference genome. Total 190 nearby genes of 195 DELs were identified. Those 190 genes were overlapped with 1321 DEMs in stage I LUAD, and 42 nearby DEMs of 38 DELs were available, as Figure 2 shown. IRF6, DIEXF and LAMB3 were the nearby DEMs of LOC101930114.

\section{DEL/DEM co-expression network construction}

In order to investigate the potential functions of DELs, DEL/DEM co-expression network was constructed. PCC of each DEL-DEM co-expression pair among 175 DELs and 1321 DEMs was calculated based on the expression level of DELs/DEMs. 
Table 1: Top 15 up- and down-regulated DELs in stage I LUAD

\begin{tabular}{|c|c|c|c|c|}
\hline IncRNAs & Chromosome & Position & $\log _{2} \mathrm{FC}$ & $P$-value \\
\hline \multicolumn{5}{|l|}{ Up-regulation } \\
\hline LOC 80078 & chr6 & $79307668-79313384$ & 8.959 & 0.02875 \\
\hline LOC 105371855 & $\operatorname{chr} 17$ & $62701313-62808381$ & 6.49313 & 0.0358 \\
\hline HNF1A-AS1 & $\operatorname{chr} 12$ & $120969837-120972292$ & 6.30907 & 0.0176 \\
\hline LINC00858 & $\operatorname{chr} 10$ & $84279979-84294659$ & 5.68535 & 0.0238 \\
\hline LOC 101926969 & $\operatorname{chr} 2$ & $91571574-91580863$ & 4.86016 & 0.0358 \\
\hline LOC442497 & $\operatorname{chr} 7$ & $379424-382879$ & 4.42594 & 0.0328 \\
\hline LOC 105374398 & $\operatorname{chr} 4$ & $35981204-35985266$ & 4.26679 & 0.02985 \\
\hline LOC 105371082 & $\operatorname{chr} 16$ & $11249045-11523533$ & 3.95453 & 0.0459 \\
\hline RAB30-AS1 & $\operatorname{chr} 11$ & $83072065-83073712$ & 3.42599 & 0.0424 \\
\hline CDKN2B-AS1 & $\operatorname{chr} 9$ & $21967138-22121097$ & 3.11379 & 0.0315 \\
\hline LOC 105370333 & $\operatorname{chr} 13$ & $100086227-100088973$ & 3.04753 & 0.04915 \\
\hline LOC 105374994 & chr6 & $26813826-26827989$ & 3.03417 & 0.0422 \\
\hline LOC 105375172 & $\operatorname{chr} 7$ & 17374916-17558909 & 2.79503 & 0.00005 \\
\hline LINC01106 & $\operatorname{chr} 2$ & $110375108-110384843$ & 2.73542 & 0.0033 \\
\hline HAGLR & $\operatorname{chr} 2$ & $176173188-176190907$ & 2.03736 & 0.00005 \\
\hline \multicolumn{5}{|l|}{ Down-regulation } \\
\hline LOC101930114 & $\operatorname{chr} 1$ & $209661358-209784545$ & -5.71679 & 0.0011 \\
\hline LOC 102724660 & $\operatorname{chr} 7$ & $128434062-128469400$ & -5.61929 & 0.04095 \\
\hline LOC 105369761 & $\operatorname{chr} 12$ & $49539040-49568225$ & -5.43482 & 0.0215 \\
\hline LOC100507487 & $\operatorname{chr} 4$ & $128428015-128519398$ & -5.00157 & 0.04675 \\
\hline TUSC7 & $\operatorname{chr} 3$ & $116709787-116717040$ & -4.69494 & 0.0344 \\
\hline LOC 105378016 & chr6 & $136557045-136793051$ & -4.6798 & 0.04305 \\
\hline NR2F2-AS1 & $\operatorname{chr} 15$ & $96127359-96340263$ & -4.62638 & 0.03075 \\
\hline LOC101928926 & $\operatorname{chr} 19$ & $19756370-19791747$ & -4.29708 & 0.0107 \\
\hline LOC 105371720 & $\operatorname{chr} 17$ & $30236905-30292166$ & -4.11397 & 0.03245 \\
\hline LOC 105370818 & $\operatorname{chr} 15$ & $51915876-51971801$ & -4.10768 & 0.00825 \\
\hline FAM95B1 & $\operatorname{chr} 9$ & $40323570-40329220$ & -3.95732 & 0.0243 \\
\hline SALRNA2 & $\operatorname{chr} 15$ & $70635248-70637314$ & -3.88695 & 0.0369 \\
\hline LOC101930496 & $\operatorname{chr} 17$ & $83110232-83119423$ & -3.59853 & 0.03035 \\
\hline LOC284865 & $\operatorname{chr} 22$ & 20198729-20204918 & -3.58415 & 0.0288 \\
\hline LOC 105370168 & $\operatorname{chr} 13$ & $39208770-39227906$ & -3.57939 & 0.0424 \\
\hline
\end{tabular}

DELs: differentially expressed long non-coding RNAs; LUAD: lung adenocarcinoma; FDR: false discovery rate; FC: fold change; chr: chromosome.

DEL-DEM co-expression pairs with $|\mathrm{PCC}>0.9|$ was subjected to network construction. In Figure 3 shown, CDKN2B-AS1, FENDRR, LINC00312, LINC00515 and LINC00162 co-expressed with 105, 63, 61, 6 and 5 DEMs, respectively.

\section{DEL-DEMI-DEM network}

In our study, DEL-DEMI-DEM network were constructed. As Figure 4 shown, DEMI-DEM interaction pairs and the nearby DEMs of DELs were depicted in 
Table 2: Top 15 up- and down-regulated DEMs in stage I LUAD

\begin{tabular}{|c|c|c|c|c|}
\hline mRNAs & Chromosome & Position & $\log _{2} \mathrm{FC}$ & $P$-value \\
\hline \multicolumn{5}{|l|}{ Up-regulation } \\
\hline EEF1A2 & $\operatorname{chr} 20$ & $63488011-63499315$ & 7.95929 & 0.0456 \\
\hline CEACAM5 & chr19 & $41708610-41730433$ & 6.26554 & 0.0002 \\
\hline CLDN2 & $\operatorname{chrX}$ & $106900062-106930861$ & 5.83291 & 0.00005 \\
\hline HABP2 & $\operatorname{chr} 10$ & $113550830-113664070$ & 5.77961 & 0.01665 \\
\hline COL11A1 & chr1 & $102876466-103108576$ & 5.49743 & 0.008 \\
\hline SLC9A2 & $\operatorname{chr} 2$ & $102619688-102711350$ & 5.29808 & 0.0217 \\
\hline PTPRZ1 & $\operatorname{chr} 7$ & $121873104-122062036$ & 5.25139 & 0.00325 \\
\hline MMP13 & $\operatorname{chr} 11$ & $102942991-102955734$ & 5.02238 & 0.01595 \\
\hline LOC100129940 & $\operatorname{chr} 12$ & $65758019-65966291$ & 4.97897 & 0.0378 \\
\hline TOX3 & $\operatorname{chr} 16$ & $52436424-52547802$ & 4.77916 & 0.0247 \\
\hline ETV4 & $\operatorname{chr} 17$ & $43527842-43546432$ & 4.73691 & 0.0109 \\
\hline HS6ST2 & $\operatorname{chrX}$ & $132626009-132961395$ & 4.63563 & 0.01275 \\
\hline MMP7 & $\operatorname{chr} 11$ & $102520507-102530747$ & 4.58386 & 0.00005 \\
\hline DKK1 & $\operatorname{chr} 10$ & $52314280-52317657$ & 4.30752 & 0.04615 \\
\hline TMPRSS4 & $\operatorname{chr} 11$ & $118015771-118125505$ & 4.26309 & 0.0133 \\
\hline \multicolumn{5}{|l|}{ Down-regulation } \\
\hline ANKRD1 & chr10 & $90912099-90921275$ & -7.62319 & 0.04905 \\
\hline TRIM58 & $\operatorname{chr} 1$ & $247857198-247880138$ & -5.23987 & 0.0444 \\
\hline CST6 & $\operatorname{chr} 11$ & $66011990-66013505$ & -4.39682 & 0.0237 \\
\hline SLC5A4 & $\operatorname{chr} 22$ & $32218420-32353008$ & -4.2162 & 0.0051 \\
\hline SLC6A4 & $\operatorname{chr} 17$ & $30194318-30235968$ & -4.11118 & 0.00025 \\
\hline MGAT5B & $\operatorname{chr} 17$ & $76850502-76950393$ & -3.92805 & 0.0085 \\
\hline TMEM100 & $\operatorname{chr} 17$ & $55719626-55732121$ & -3.91121 & 0.00025 \\
\hline LOC 105375355 & $\operatorname{chr} 7$ & $76510101-76544090$ & -3.84847 & 0.03815 \\
\hline ADGRE2 & $\operatorname{chr} 19$ & $14689786-14778541$ & -3.67241 & 0.00015 \\
\hline KIF19 & $\operatorname{chr} 17$ & $74326211-74355820$ & -3.50716 & 0.0445 \\
\hline DTHD1 & $\operatorname{chr} 4$ & $36281610-36345756$ & -3.34849 & 0.037 \\
\hline FCN3 & $\operatorname{chr} 1$ & $27369109-27374825$ & -3.32501 & 0.0024 \\
\hline ZMYND10 & $\operatorname{chr} 3$ & $50341105-50346028$ & -3.28484 & 0.0329 \\
\hline BTNL9 & $\operatorname{chr} 5$ & $181040224-181061523$ & -3.03646 & 0.0001 \\
\hline VIPR1 & $\operatorname{chr} 3$ & $42489298-42537573$ & -3.00424 & 0.00005 \\
\hline
\end{tabular}

DEMs: differentially expressed mRNAs; LUAD: lung adenocarcinoma; FDR: false discovery rate; FC: fold change; chr: chromosome.

the network. For instance, in Figure 4, FOXF1 was the nearby DEM of lncRNA FENDRR and were negatively regulated by hsa-miR-200b-3p and hsa-miR-439. CDKN2B was the nearby DEM of lncRNA CDKN2BAS1 and was negatively targeted by hsa-miR-182-5p and hsa-miR-375.

\section{GO and KEGG pathway enrichment}

1321 DEMs were significantly enriched in pathways in cancer (Kegg:05200), cell adhesion molecules(Kegg:04514), focal adhesion (Kegg:04510), cytokine-cytokine receptor interaction (Kegg:04060), 


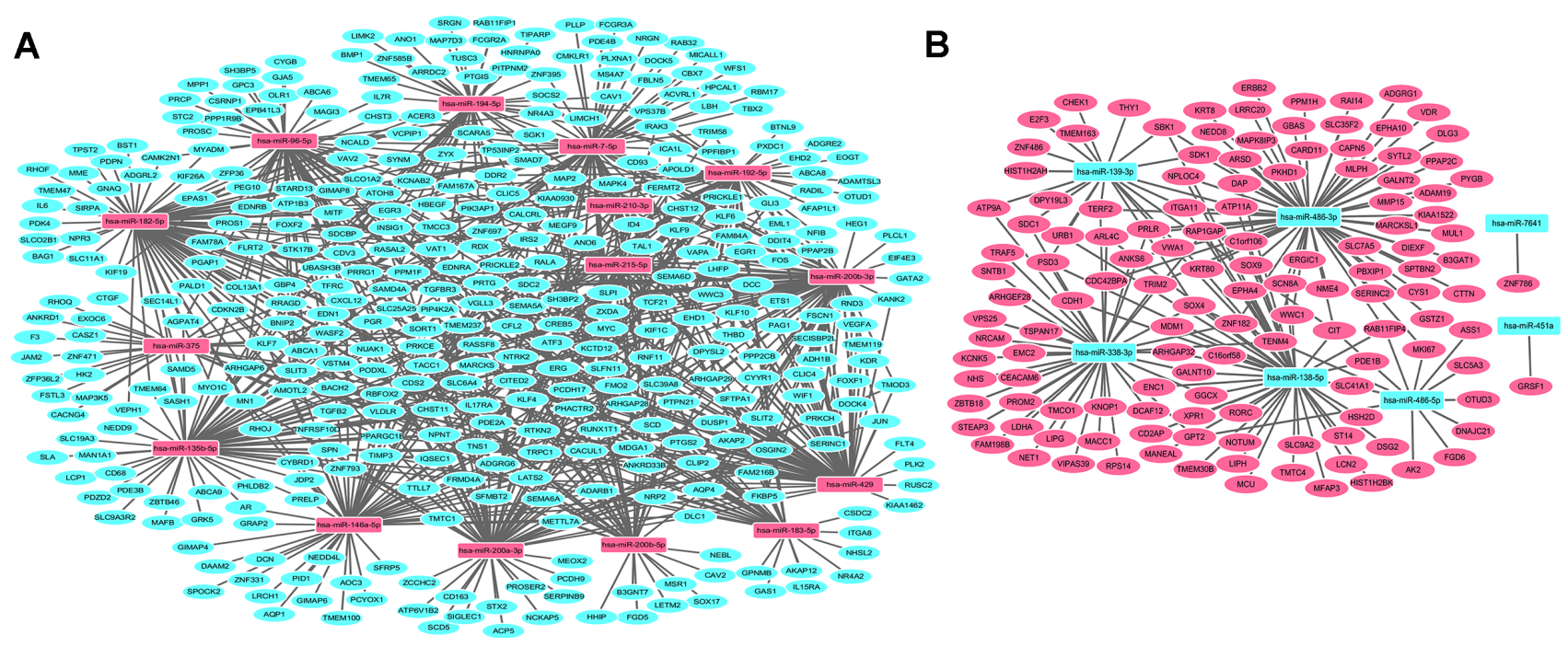

Figure 1: DEMI-DEM regulatory network in stage I lung adenocarcinoma. (A) The network among up-regulated DEMIs and down-regulated DEMs. (B) The network among down-regulated DEMIs and up-regulated DEMs. The rectangle node and circular node indicated DEMIs and DEMs, respectively; the rose color and turquoise color represented up-regulation and down-regulation, respectively. DEMIs indicated differentially expressed microRNAs; DEMs were differentially expressed protein-coding mRNAs.

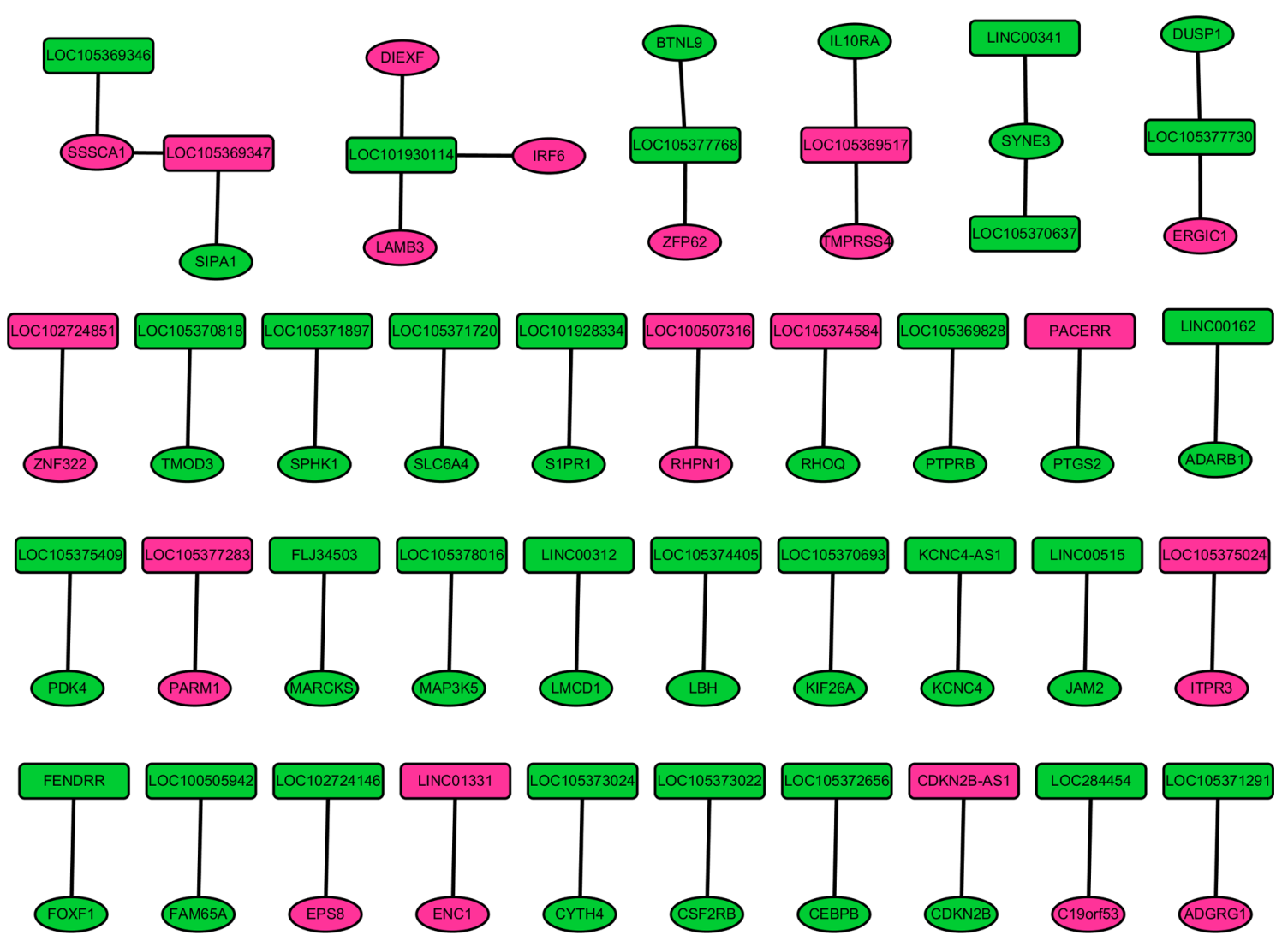

Figure 2: The nearby DEMs of DELs in stage I lung adenocarcinoma. The rectangle and circular nodes indicated DELs and DEMs, respectively; rosecolor and green color indicated up- and down-regulation, respectively. DELs indicated differentially expressed long non-coding RNAs; DEMs were differentially expressed protein-coding mRNAs. 
ECM-receptor interaction (Kegg:04512) and tight junction (Kegg:04530) (Table 3). Furthermore, those DEMs were obviously enriched in signal transduction, cell adhesion, angiogenesis, regulation of cell proliferation, regulation of cell migration, regulation of cell apoptosis and response to hypoxia of Gene Ontology (GO) biological process (Table 3).

\section{qRT-PCR validation of the expression levels of dysregulatedDEMIs/DEMs in stage I LUAD}

qRT-PCR was subjected to validate the expression levels of dysregulated DEL/DEMI/DEM in 6 stage I LUAD tissues and 6 adjacent non-tumor tissues. 3 DELs, 5 DEMIs and 6 DEMs were used to apply for qRT-PCR verification. As Figure 5A-5E shown, hsa-miR-200a-3p $(P<0.01)$, hsa-miR-200b-3p $(P<0.05)$, hsa-miR-200b-5p $(P<0.05)$, hsa-miR-200c-5p $(P<0.05)$ and hsa-miR-429 $(P<0.01)$ were obviously up-regulated in stage I LUAD tissues and hsa-miR-338-3p $(P<0.05)$ was obviously down-regulated in stage I LUAD tissues compared with adjacent non-tumor tissues. The expression levels of ADARB1 $(P<0.01)$, ADRB2 $(P<0.05)$ and ANKRD1 $(P<0.05)$ were significantly down-regulated in stage I LUAD tissues; COL1A1 $(P<0.05)$ and MMP13 $(P<0.05)$ were significantly up-regulated in stage I LUAD tissues compared with adjacent non-tumor tissues (Figure 5G$5 \mathrm{~K})$.

\section{The expression levels of candidate DELs in LUAD were analyzed based on TCGA datasets and qRT-PCR experiments}

The lncRNA expression profiling of LUAD tissues (case group) and adjacent non-tumor tissues (control group) were retrieved from TCGA database. In addition, the expression of 12 candidate DELs in LUAD tissues was detected through qRT-PCR methods in 6 stage I LUAD tissues and 6 adjacent non-tumor tissues. As Figure 6A and $6 \mathrm{E}$ shown, both of lncRNA MINCR (Figure 6A) and LBX2-AS1 (Figure 6E) weresignificantlyup-regulated in LUAD tissues based on TCGA dataset, however,
A
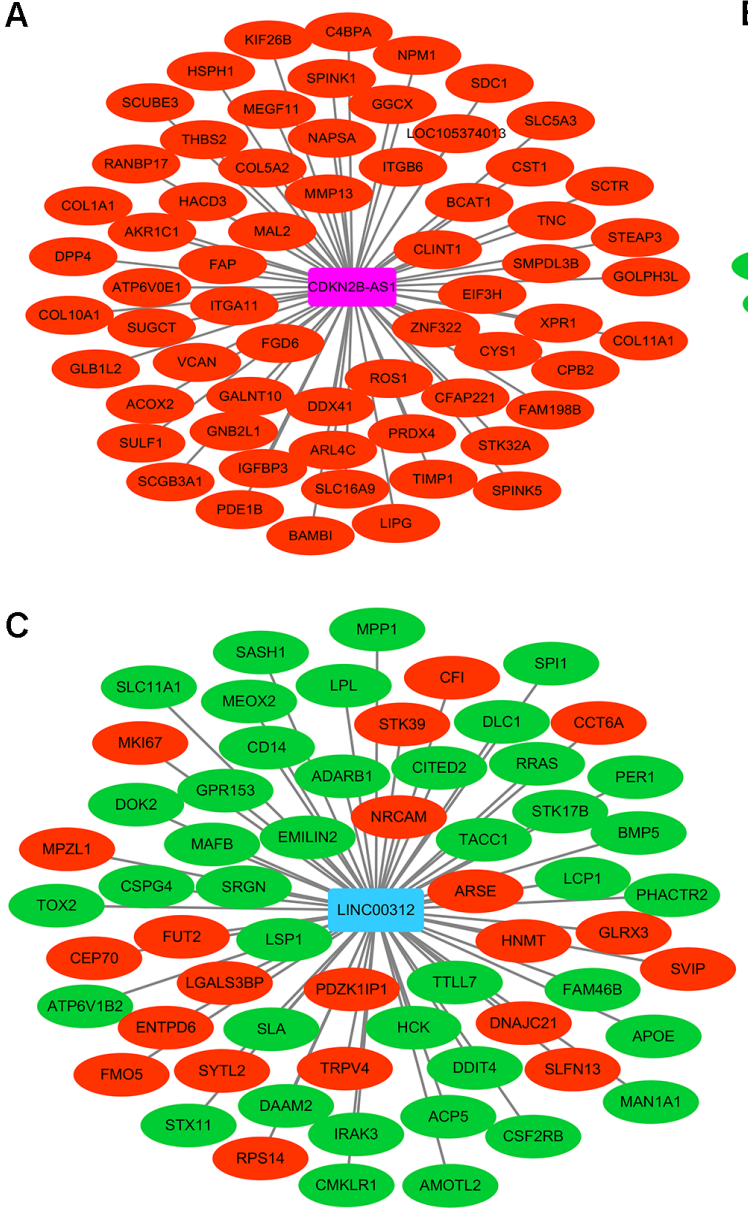

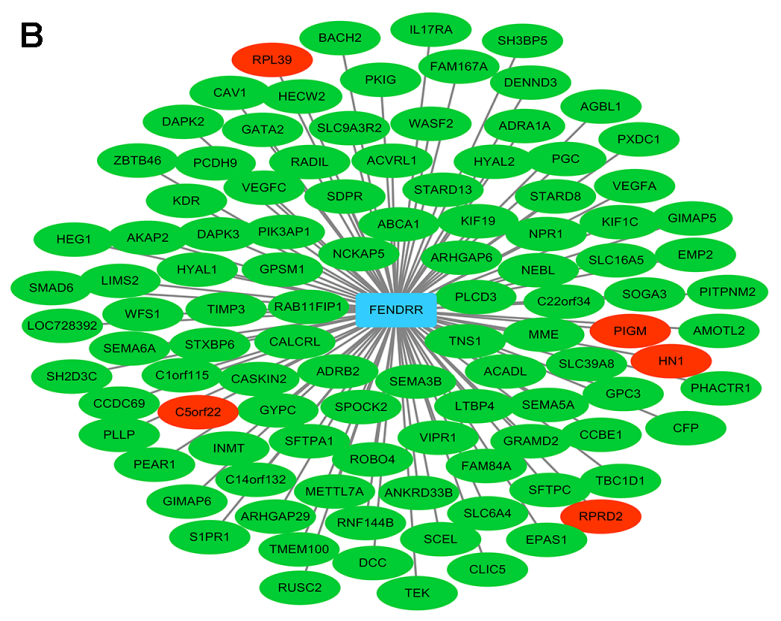

E

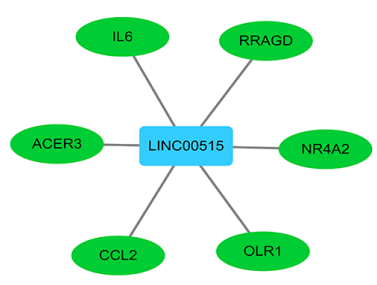

$\mathbf{F}$

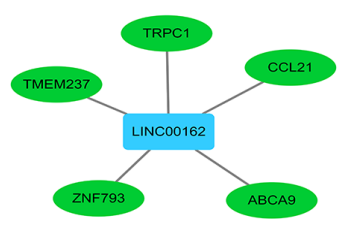

Figure 3: Co-expression network among DELs and DEMs in stage I lung adenocarcinoma. (A) Sub-network of CDKN2BAS1; (B) sub-network of FENDRR; (C) sub-network of LINC00312; (D) sub-network of LINC00515; (E) sub-network of LINC00162. Rectangle nodes and circular nodes represented DELs and DEMs, respectively. Rose color and red color indicated up-regulation; blue color and green color indicated down-regulation. 
thosetwo lncRNAweresignificantly down-regulated in LUAD tissues based on qRT-PCR results. In Figure 6B, 6C, 6D and 6F, LINC00963 (Figure 6B), NR2F2-AS1 (Figure 6C), LINC00515 (Figure 6D), and LINC00162 (Figure 6F) were significantly down-regulated in LUAD tissues both in TCGA dataset and qRT-PCR experiment. As Supplementary Figure 2A-2D shown, LINC00312 (Supplementary Figure 2A), MGC27382 (Supplementary Figure 2B), LINC00472 (Supplementary Figure 2C) and FENDRR (Supplementary Figure 2D) were significantly down-regulated in LUAD tissues both inTCGA dataset and qRT-PCR experiment. CDKN2B-AS1 was significantly up-regulated in LUAD tissues based on TCGA dataset and it had the up-regulated tendency in LUAD tissues based on qRT-PCR (Supplementary Figure 2E). HNF1A-AS1 was significantly up-regulated in LUAD tissues based on TCGA dataset and had the down-regulated tendency in LUAD tissues based on qRT-PCR results (Supplementary Figure 2F).

Combined with the bioinformatics analyses of our RNA-sequencing, the expression 8 of 12 candidate lncRNAs including LINC00963, NR2F2-AS1, LINC00515, LINC00162, LINC00312, MGC27382, LINC00472 and FENDRR were significantly up-regulated in LUAD tissues based on our RNA-sequencing, TCGA dataset and qRT-PCR experiment. CDKN2B-AS1 was significantly up-regulated in LUAD tissues based on our
RNA-sequencing and TCGA dataset, and it had the upregulated tendency in LUAD tissues based on qRT-PCR results. MINCR and LBX2-AS1 were significantly upregulated in LUAD tissues both in our RNA-sequencing and TCGA dataset and those two lncRNA was significantly down-regulated in LUAD tissues based on qRT-PCR. HNF1A-AS1 was significantly down-regulated in LUAD tissues both in our RNA-sequencing and qRT-PCR, and it is significantly up-regulated in LUAD tissues based on TCGA dataset.

In summary, the expression levels of 10 of 12 DELs in LUAD through qRT-PCR experiments were compatible with our RNA-sequencing; and the expression levels of 9 of 12 DELs in LUAD tissues through qRT-PCR experiments were compatible with the TCGA dataset. In general, the results of cross validation indicated that the expression patterns of candidate DELs in LUAD based on TCGA database and qRT-PCR was compatible with our RNA-sequencing and bioinformatics analyses.

\section{Receiver operating characteristic (ROC) curve analysis}

In order to assess the discriminatory ability of the 12 candidate DELs among LUAD tissues and adjacent nontumor tissues generated from TCGA database, ROC curve analyses were conducted and area under the curve (AUC)

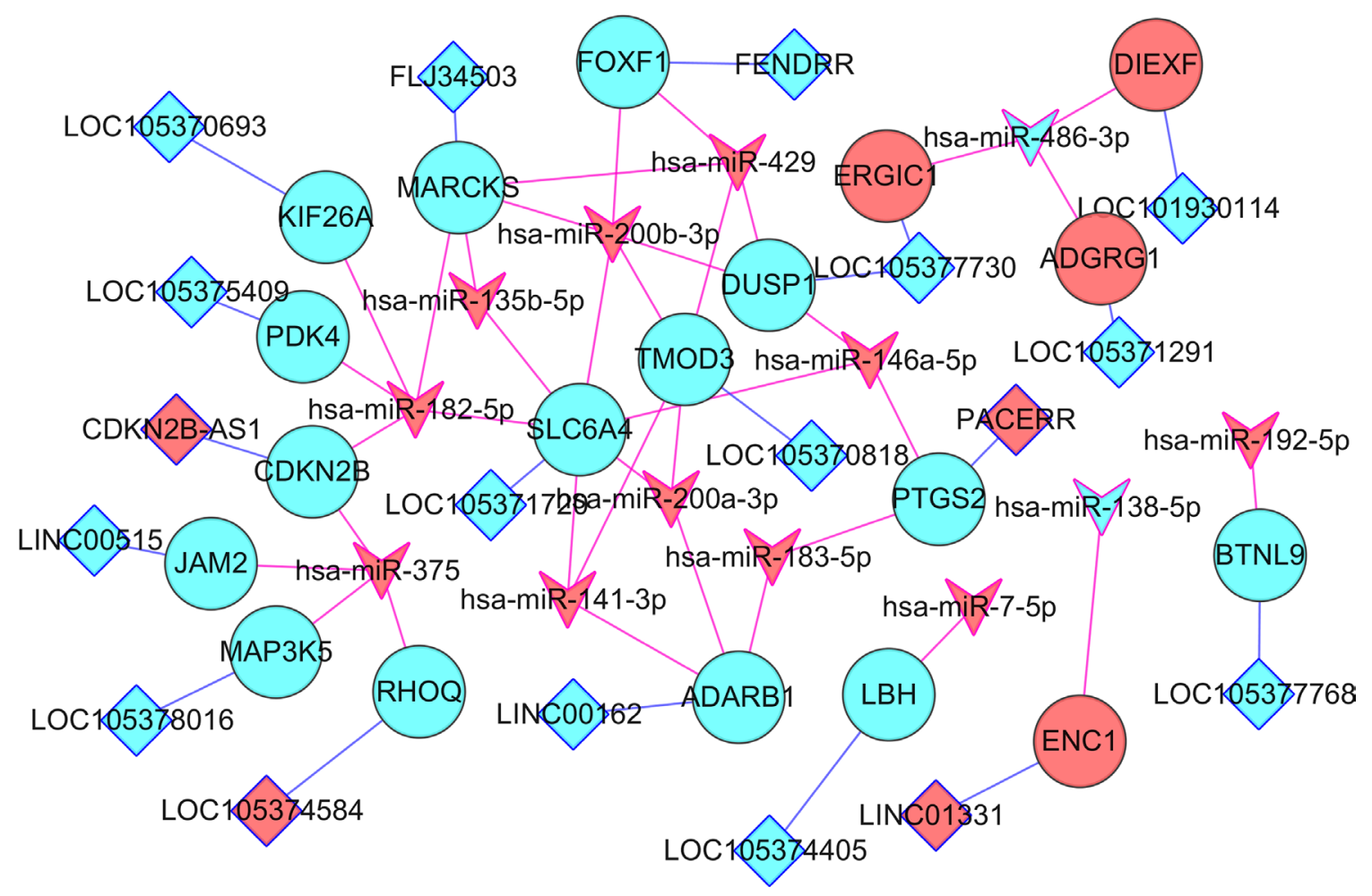

Figure 4: DEL-DEMI-DEM network. Diamond nodes and circular nodes indicated DELs and DEMs, respectively. V nodes represented DEMIs. Blue line indicated the nearby link between DEL and DEM; red line indicated the negative correlation between DEMI and DEM. Red color and turquoise color was up- and down-regulation. DELs indicated differentially expressed long non-coding RNAs; DEMs were differentially expressed protein-coding mRNAs; DEMIs indicated differentially expressed microRNAs. 
Table 3: GO biological process and KEGG signaling pathway enrichment of DEMs in stage I LUAD

\begin{tabular}{|c|c|c|c|}
\hline Items & Details & Support genes & FDR \\
\hline \multicolumn{4}{|c|}{ GO-biological process enrichment (top 15) } \\
\hline GO:0007165 & Signal transduction & 135 & $2.90 \mathrm{E}-29$ \\
\hline GO:0007155 & Cell adhesion & 73 & $2.74 \mathrm{E}-18$ \\
\hline GO:0001525 & Angiogenesis & 35 & $6.29 \mathrm{E}-15$ \\
\hline GO:0007275 & Multicellular organismal development & 89 & $1.89 \mathrm{E}-13$ \\
\hline GO:0008284 & Positive regulation of cell proliferation & 47 & $2.08 \mathrm{E}-11$ \\
\hline GO:0007596 & Blood coagulation & 53 & $4.95 \mathrm{E}-11$ \\
\hline GO:0030335 & Positive regulation of cell migration & 24 & $1.37 \mathrm{E}-10$ \\
\hline GO:0001666 & Response to hypoxia & 30 & $4.56 \mathrm{E}-10$ \\
\hline GO:0030154 & Cell differentiation & 55 & 4.89E-10 \\
\hline GO:0007275 & Multicellular organismal development & 44 & $6.01 \mathrm{E}-10$ \\
\hline GO:0042493 & Response to drug & 38 & $5.85 \mathrm{E}-09$ \\
\hline GO:0043066 & Negative regulation of apoptotic process & 35 & $1.76 \mathrm{E}-08$ \\
\hline GO:0001501 & Skeletal system development & 24 & $1.81 \mathrm{E}-08$ \\
\hline GO:0008285 & Negative regulation of cell proliferation & 39 & $5.06 \mathrm{E}-08$ \\
\hline GO:0009612 & Response to mechanical stimulus & 14 & $5.52 \mathrm{E}-08$ \\
\hline \multicolumn{4}{|c|}{ KEGG pathway enrichment (top 15) } \\
\hline Kegg:05200 & Pathways in cancer & 37 & 2.92E-07 \\
\hline Kegg:04514 & Cell adhesion molecules (CAMs) & 20 & $2.02 \mathrm{E}-06$ \\
\hline Kegg:04510 & Focal adhesion & 26 & $2.30 \mathrm{E}-06$ \\
\hline Kegg:04060 & Cytokine-cytokine receptor interaction & 30 & 2.64E-06 \\
\hline Kegg:04512 & ECM-receptor interaction & 16 & 3.19E-06 \\
\hline Kegg:04360 & Axon guidance & 19 & 8.68E-06 \\
\hline Kegg:04610 & Complement and coagulation cascades & 13 & $1.94 \mathrm{E}-05$ \\
\hline Kegg:04144 & Endocytosis & 23 & 2.17E-05 \\
\hline Kegg:04380 & Osteoclast differentiation & 18 & $2.25 \mathrm{E}-05$ \\
\hline Kegg:05144 & Malaria & 11 & $2.52 \mathrm{E}-05$ \\
\hline Kegg:04530 & Tight junction & 18 & $2.76 \mathrm{E}-05$ \\
\hline Kegg:04666 & Fc gamma R-mediated phagocytosis & 15 & $2.82 \mathrm{E}-05$ \\
\hline Kegg:04350 & TGF-beta signaling pathway & 14 & $3.09 \mathrm{E}-05$ \\
\hline Kegg:05219 & Bladder cancer & 9 & 0.000171 \\
\hline Kegg:05140 & Leishmaniasis & 8 & 0.00027 \\
\hline
\end{tabular}

GO: gene ontology; KEGG: Kyoto Encyclopedia of Genes and Genomes; FDR: false discovery rate; DEMs: differentially expressed mRNAs; LUAD: lung adenocarcinoma. 
were calculated. As Figure 7A-7K shown, the AUC of 10 DELs was more than 0.7. The AUC of CDKN2B-AS1 and HNF1A-AS1 was respective 0.563 and 0.529 , less than 0.7 (Figure 7L, 7K). LINC00312, MGC27382, LINC00472 and FENDRR had the largest AUC in those 12 DELs. For LUAD diagnosis, the sensitivity and specificity of LINC00312 was 93.1\% and 82.8\% (Figure 7G); the sensitivity and specificity of MGC27382 was $96.6 \%$ and $91.8 \%$ (Figure $7 \mathrm{H}$ ); the sensitivity and specificity of LINC00472 was 91.4\% and 93.9\% (Figure 7I); the sensitivity and specificity of FENDRR was $96.6 \%$ and 92.4\% (Figure 7J); respectively.

\section{DISCUSSION}

In our study, all of the members of miR-200 family, such as miR-200a,-3p, miR-200a-5p, miR-200b-5p, miR200b-3p, miR-200c-5p, miR-429, miR-141-5p and miR200c-5p were obviously up-regulated (more than 2 fold) in stage I LUAD tissues compared to adjacent non-tumor tissue, which was accordance with the previous study [9]. The members of miR-200 family are the powerful regulator of epithelial-to-mesenchymal transition, which is essential in cell adhesion, cell invasion and cell metastasis in non-small cell lung cancer [9-12]. Increased miR-429

promotes cell proliferation and cell metastasis in NSCLC [13]. In addition to, serum levels of miR-141, miR-200b and miR-429 are potential biomarkers for early diagnosis in lung cancer $[14,15]$.

Recent evidence highlights lncRNA as vital regulators of cancer biology that contribute to cancer cell functions including cell proliferation, cell apoptosis, and metastasis. In our study, total of 75 DELs were identified in stage I LUAD.

LncRNA CDKN2B-AS1 (CDKN2B antisense RNA1, also named as ANRIL), a 3.8-kb-long RNA, transcribed from the short arm of human chromosome 9 on p21.3, was significantly up-regulated in stage I LUAD tissues compared to adjacent non-tumor tissues, which was consistent with the published article [16]. It is reported that CDKN2B-AS1 genetic polymorphisms are significantly associated with lung cancer susceptibility and response to platinum-based chemotherapy [17]. CDKN2B-AS1 is increased in lung cancer plasma samples, lung cancer tissues and lung cancer cell lines compared with those samples in healthy volunteers, adjacent non-tumor tissues and normal human bronchial epithelial cells [18]. Higher expression of CDKN2B-AS1 is significantly correlated with higher TNM stage, larger tumor size and shorter overall survival in patients with NSCLC [16].CDKN2B
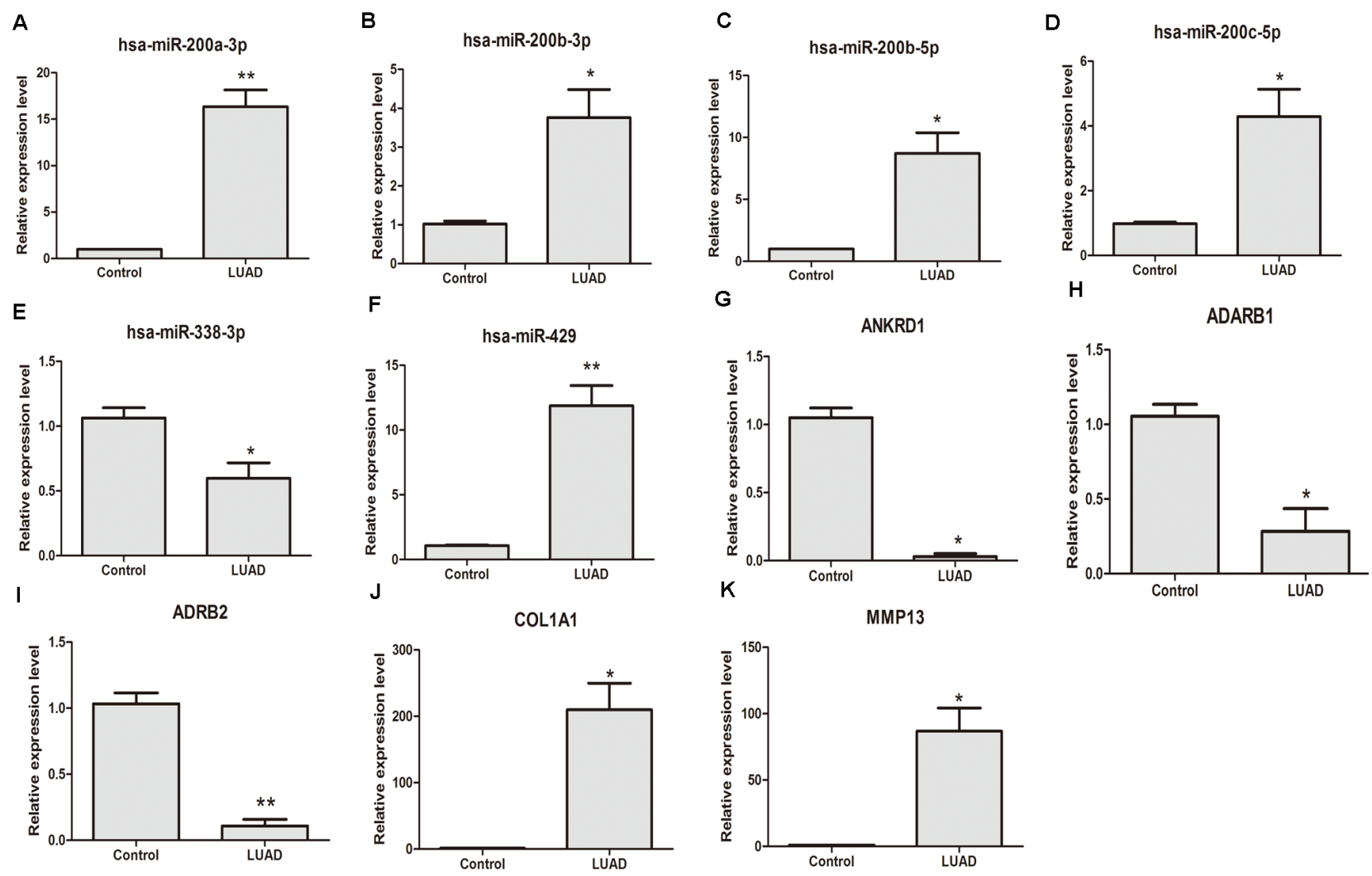

Figure 5: qRT-PCR validation of dysregulated DEMIs/DEMs in stage I LUAD compared with adjacent non-tumor tissues. (A) hsa-miR-200a-3p; (B) hsa-miR-200b-3p; (C) hsa-miR-200b-5p; (D) hsa-miR-200c-5p; (E) hsa-miR-338-5p; (F) hsa-miR-428; (G) ADARB1; (H) ADRB2; (I) ANKRD1; (J) COL1A1; (K) MMP13. LUAD indicated stage I lung adenocarcinoma tissues; control indicated paired adjacent non-tumor tissues. * represented $P<0.05$ and ** represented $P<0.01$. 

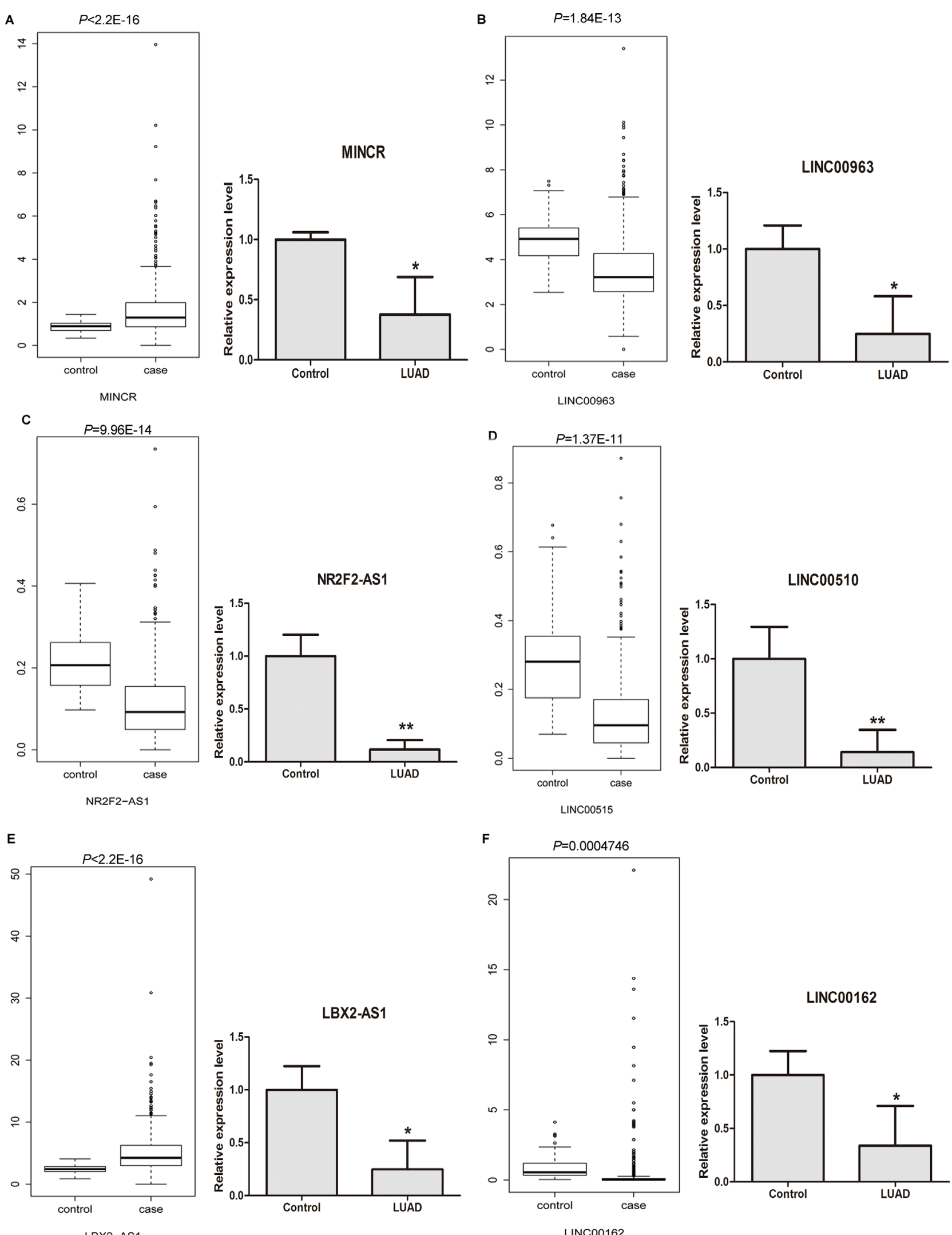

Figure 6: The cross validation of the expression levels of candidate DELs in LUAD tissues based on TCGA database and qRT-PCR experiments. (A) MINCR; (B) LINC00963; (C) NR2F2-AS1; (D) LINC00515; (E) LBX2-AS1; (F) LINC00162. Case group and control group indicated LUAD tissues and adjacent non-tumor tissues, respectively. LUAD indicated lung adenocarcinoma tissues. * represented $P<0.05$ and ** represented $P<0.01$. 
encodes a cyclin-dependent kinase inhibitor and functions as the regulator of cell growth. CDKN2B is the nearby DEM of CDKN2B-AS1 and was obviously downregulated in stage I LUAD tissues compared with adjacent non-tumor tissues. Copy number loss of CDKN2B predicts poor survival in patients with lung squamous cell carcinoma [19]. CDKN2B deficiency accelerates mutant KRAS lung tumorigenesis in mice mode, which leads to cell metastasis and cell proliferation [20]. In the DEL-DEM network, CDKN2B-AS1 co-expressed 63 upregulated DEMs, such as MMP13, COL11A1, COL1A1, ITGA11, VACN, THBS2 and TIMP1, which were significantly enriched in cell adhesion molecules and focal adhesion pathways and enriched in cell adhesion, response to hypoxia, signal transduction and positive regulation of cell proliferation of GO biological process. In our previous study, THBS2, COL11A1 and VACN were also identified as up-regulated genes in NSCLC compared with adjacent non-tumor tissues through integrated analysis of microarray data [21]. Markedly over-expression of THBS2 and its co-expressed genes (such as VCAN, CLO11A1,
FAP) predicts poor survival of patients with lung cancer; down-regulation of VCAN and THBS2 inhibits cell proliferation in NSCLC [22].COL11A1 and COL1A1 belong to fibrillar collagen. COL11A1 is over-expressed in NSCLC with lymph node metastasis and recurrent NSCLC tissues, which promotes cell proliferation, cell migration, cell invasion and chemo-resistance [23]. The biological roles of COL1A1 in LUAD are unknown. Ectopic expression of MMP13 (matrix metallopeptidase 13) predicts poorer-5-year survival in patients with NSCLC [24].

In the DEL-DEM co-expression network, FENDRR (also named as FOXF1-AS1)had the high connectivity with DEMs, which co-expressed with 105 DEMs. Those DEMs were significantly enriched in pathways in cancer, focal adhesion and cytokine-cytokine receptor interaction pathways; and enriched in angiogenesis, regulation of cell proliferation, cell adhesion and signal transduction of GO biological process. FENDRR is identified as one of the most aberrantly expressed lncRNAs in human Xuanwei lung cancer through microarray analysis [25]. A recent

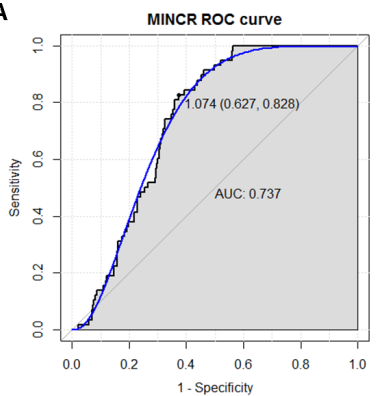

E
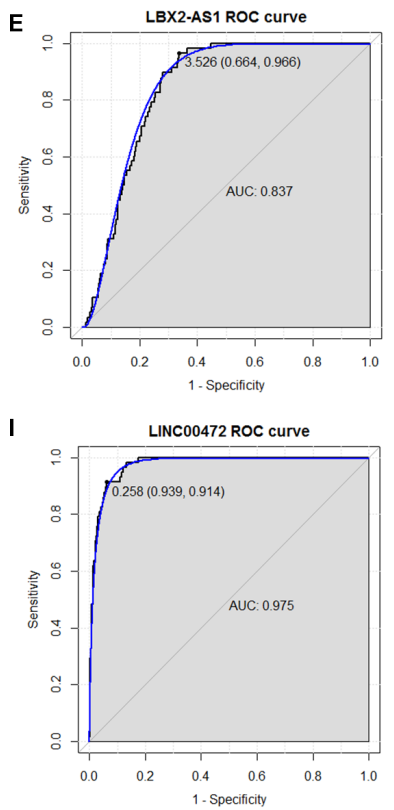
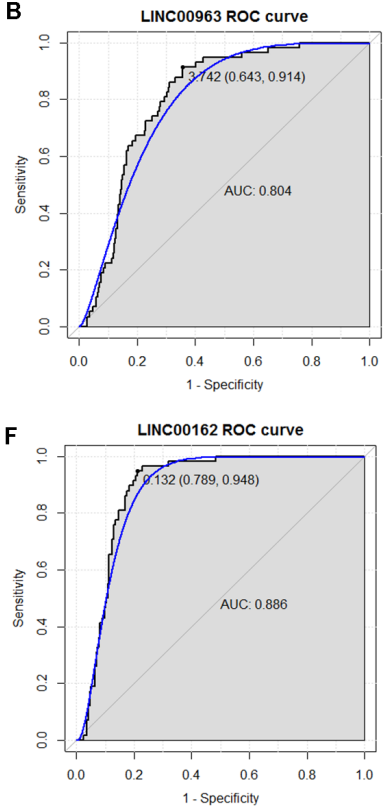

$\mathbf{J}$

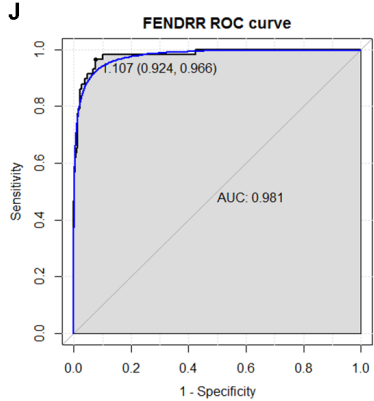

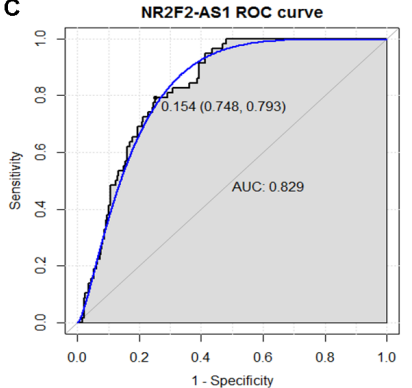

G

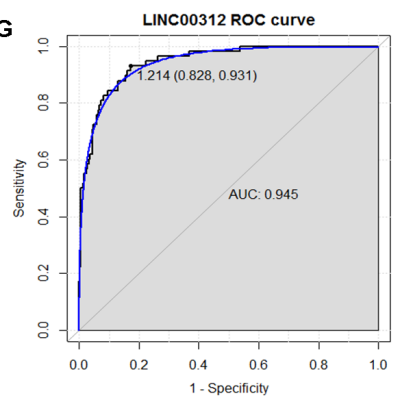

H

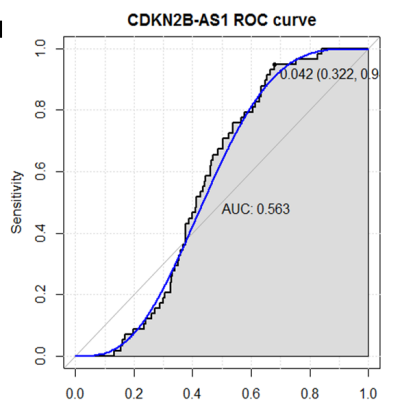

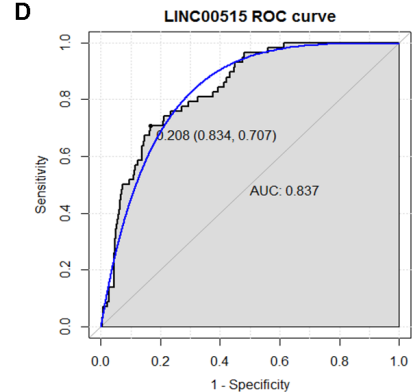

H
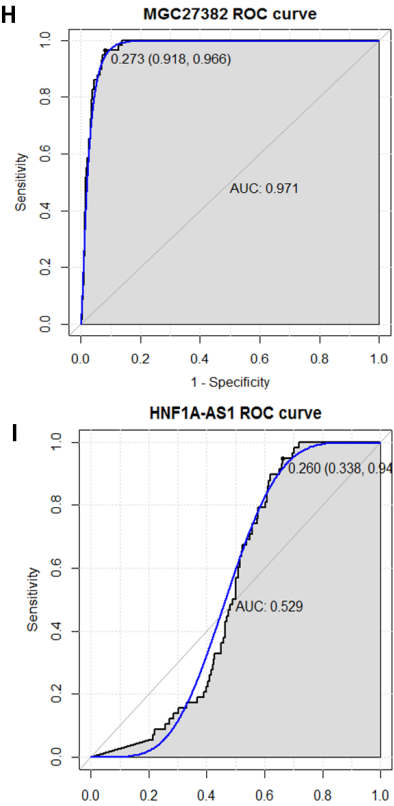

Figure 7: The discriminatory ability of DELs between LUAD tissues and adjacent non-tumor tissues was accessed with ROC curve. (A) MINCR; (B) LINC00963; (C) NR2F2-AS1; (D) LINC00515; (E) LBX2-AS1; (F) LINC00162; (G) LINC00312; (H) MGC27382; (I) LINC00472; (J) FENDRR; (K) CDKN2B-AS1; (L) HNF1A-AS1. 
published article demonstrates that over-expression of FENDRR inhibits cell migration, cell invasion and mediates stem-like properties by regulating epithelia-mesenchymal transition in NSCLC [26]. FOXF1 was the nearby DEM of FENDRR, and it is down-regulated in stage I LUAD tissues. Up-regulated FOXF1 has anti-malignant effects of mesenchymal stem cell fusion-induced reprogramming on lung cancer cells [27]. In the DEL-DEMI-DEM network, FOXF1 was negatively targeted by miR-200 family members of miR-429 and miR-200b-3p. ADRB2 was one of co-expressed genes with FENDRR and it was significantly down-regulated in stage I LUAD tissues. In our previous article, ADRB2 (encodes beta-2-adrenergic receptor, a member of the $G$ protein-coupled receptor superfamily) is also down-regulated in NSCLC tissues compared with adjacent non-tumor tissues through integrated analysis of microarray data [21]. Genetic variants in ADRB2 confer the risk of chronic obstructive pulmonary disease and lung adenocarcinoma [28, 29]. The roles of ADRB2 in cell behaviors of LUAD including cell proliferation, cell invasiveness and cell metastasis are unclear.

LINC00162, LINC00515 and LINC00312 were significantly down-regulated in stage I LUAD tissues compared with adjacent non-tumor tissues. LINC00162 is P38 inhibited cutaneous squamous cell carcinoma associated lincRNA, also named as PICSAR and NLC1-C. Knockdown of LINC00162 suppresses cell proliferation and cell migration by inhibiting ERK1/2 activity and down-regulating the expression of DUSP6 in cutaneous squamous cell carcinoma [30]. Down-regulated LINC00162 promotes cell proliferation by repressing miR320a and miR-383in testicular embrynonal carcinoma [31].ADARB1 was the nearby DEM of LINC00162 and was down-regulated in stage I LUAD tissues. The expression level of ADARB1 in lung cancer cell lines is higher than that in normal human bronchial epithelial cells; higher mRNA expression level of ADARB1 predicts a better outcome in patients with lung adenocarcinoma. In addition to, ADARB1 was co-expressed with downregulated LINC00312 (also named as NAG7). ADARB1 was targeted by miR-200 family members of miR-183$5 \mathrm{p}$ and miR-141, miR-200a-3p. Over-expression of LINC00312 inhibits cell migration and cell invasion in bladder cancer through down-regulation of miR-197-3p [32]. Lower expression of LINC00312 predicts larger tumor size and shorter overall survival in nasopharyngeal carcinoma [33]. Total of 61 dysregulated DEMs were coexpressed with LINC00312 and were enriched in tight junction, cell adhesion molecules and pathways in cancer pathways, and enriched in angiogenesis, cell adhesion and signal transduction of GO biological process. The expression of LINC00515 is decreased in cisplatinresistant cells of high-grade serous ovarian cancer [34]. JAM2 was the nearby DEM of LINC00515. JAM2 encodes junctional adhesion molecule 2 and belongs to the immunoglobulin superfamily. Increased methylation of JAM2 leads to down-regulation of JAM2, which is associated with LUAD pathogenesis [35].JAM2 was significantly enriched in cell adhesion molecules and tight junction. Based on aforementioned information, LINC00162, LINC00515 and LINC00312 might play essential roles in tumorigenesis of LUAD and the biological functions of those dysregulated DELs in cell proliferation, invasiveness, metastasis and angiogenesis of LUAD are needed to be explored in the future work.

The ROC curve analyses indicated that LINC00312and FENDRRmight be potential biomarkers for LUAD diagnosis. In the further work, larger cohort of LUAD patients should be enrolled and the potential value of those lncRNAs in LUAD is needed to be further validated in clinical practice.

In conclusion, we identified the aberrantly expressed lnRNAs, miRNAs and mRNAs in stage I LUAD. Our study indicated that those dysregulated genes including miR-200 family, CDKN2B-AS1, FENDRR, LINC00162, LINC00515, LINC00312, ADARB1 and JAM2might synergistically contributes to tumorigenesis in stage I LUAD based on complex interactions between each other through KEGG pathways and GO biological processes including cell adhesion molecules, focal adhesion, tight junction, pathways in cancer, cell adhesion, angiogenesis and regulation of cell proliferation and regulation of cell apoptosis.

There are limitations in our study. Firstly, novel dysregulated lncRNAs were identified, such as LINC00162, LINC00515 and LINC00312, however, the biological roles of those genes involved in LUAD were not further investigated. Secondly, the diagnostic value of identified lncRNAs and miRNAs is needed to be translated into clinical practice through large cohort of LUAD patients. Our study might pave the way for illumination of pathogenesis of LUAD and discovery of potential therapeutic targets and novel diagnosis biomarkers for LUAD patients.

\section{MATERIALS AND METHODS}

\section{Patients and samples}

LUAD tumor tissues and paired adjacent non-tumor tissues for RNA-sequencing were obtained from patients who underwent surgery in the Fourth Hospital of Hebei Medical University. 4 patients with TNM I stage received none of chemo- or radiotherapy before resection surgery, were enrolled into our study (from Dec 29th, 2015 to Feb 18th, 2016). The pathological type and TNM stage of patients were explicitly diagnosed by histopathology examination. The basic information of patients including gender, age and TNM stage were recorded. The details were shown in Supplementary Table 2. This work was approved by the Ethics Committee of the Fourth Hospital of Hebei Medical University and informed written consent 
was obtained from all patients. The research complied with the principles of the Declaration of Helsinki.

\section{RNA-sequencing and data preprocessing}

Total RNA of collected specimens was extracted by TRIzol reagent (Invitrogen, Carlsbad, CA, USA) for construction of cDNA libraries according to the manufacture instruction. Likewise, cDNA libraries of small RNA were constructed. Lastly, each library was conducted to IlluminaHiSeq 4000 sequencing.

The raw image data obtained from RNA-sequencing was translated into raw FASTQ sequence data. Adaptors of sequences were trimmed; nucleotides with a quality score $<20$ were trimmed from the end of the sequence; $\mathrm{N}$ base rate of raw reads more than $10 \%$ were discarded using Cutadapt 1.9.1. For microRNA (miRNA) sequencing, sequences with length less than 18 ntor more than $32 \mathrm{nt}$ were abnegated. TopHat was used to align the clean reads of long non-coding RNAs (lncRNAs) and protein coding mRNAs (mRNAs) with the human reference genome, Ensemble GRCh38 v 84 (hg19) [36]. Moreover, the alignment of miRNAs with hg19 was implemented by Bowtie. Fragments per kilobase of exon per million fragments mapped (FPKM) was used to describe the transcription abundance of lncRNAs and mRNAs, which was quantified by cuffquant and cuffnorm. miRDeep 2 was used to quantified the transcription abundance of miRNAs [37].

\section{Differentially expressed genes analysis}

The differentially expressed lncRNAs (DELs) and differentially expressed mRNA (DEMs) were identified in stage I LUAD tissues compared with adjacent nontumor tissues by Cuffdiff. $P<0.05$ and $\mid \log _{2}$ fold change $\mid>1$ was set as the cut-off of DELs and DEMs. Differentially expressed miRNA (DEMIs) in stage I LUAD tissues were filteredusing DEGseq package in $\mathrm{R}$ language. miRNAs with FDR $<0.001$ and $\mid \log _{2}$ fold change $\mid>1$ were selected as DEMIs.

\section{DEL-DEM co-expression network construction}

Pearson correlation coefficients (PCCs) indicating co-expression relationship between DELs and DEMs were calculated. DEML-DEM co-expression pairs with $|P C C \geq 0.90|$ were retained for network construction [38]. The DEL-DEM co-expression network was visualized by Cytoscape (http://cytoscape.org/) [39].

\section{Prediction of target genes of DEMIs}

miRWalk database (http://www.umm.uniheidelberg.de/apps/zmf/mirwalk/), was used to predict the target-genes of DEMIs [40]. In our study, 6 algorithms including RNA22, miRanda, miRDB, miRWalk, PICTAR and Targetscan in miRWalk was used. Moreover, the genes, predicted by more than 4 out of 6 algorithms, were considered as the target-gene of DEMIs. The targetgenes were overlapped with DEMs in stage I LUAD and DEMI-DEMs interaction pairs in a negative manner were subjected to construct network, and visualized by Cytoscape [39].

\section{DEL-DEMI-DEM network}

First, The nearby protein-coding genes of DELs with distance $<100 \mathrm{~kb}$ were identified in GRCH38 reference genome. Those protein-coding genes of DELs were overlapped with DEMs and the nearby DEMs of DELs were identified. Then, the nearby DEMs of DELs were overlapped with those DEMs negatively targeted by DEMIs. Lastly, DEL-DEMI-DEM network was constructed and visualized by Cytoscape [39].

\section{Gene ontology and KEGG enrichment analysis}

In order to understand the potential biological functions and signaling pathways of DELs in stage I LUAD, GeneCoDis3 (http://genecodis.cnb.csic.es/ analysis) analysis were conducted to Gene Ontology (GO) biological process and Kyoto Encyclopedia of Genes and Genomes (KEGG) pathway enrichment [41]. Those Items with $\mathrm{FDR}<0.05$ was filtered as significant enrichments.

\section{Quantitative real-time polymerase chain reaction (qRT-PCR)}

Total RNA of TNM stage I LUAD tissues and adjacent non-tumor tissues were extracted by using Trizol (Invitrogen, Carlbad, CA, USA) according to the manufacture instructions. FastQuantcDNA and miRcute Plus (Tiangen, Beijing, China) and miRNA First-Strand cDNA Synthesis Kit (Tiangen, Beijing, China) was used to synthesize the cDNA of mRNA and miRNA, respectively. qRT-PCRreactions were performed by using SuperRealPreMix Plus SYBR Green Kit (Tiangen, Beijing, China) and miRcute Plus miRNAqPCR Detection Kit (Tiangen, Beijing, China) on Applied Biosystems 7500 (AppliedBiosystems, Foster City, CA, USA). GAPDH and U6 were used as internal control for mRNA and miRNA detection, respectively. The relative expression of candidate genes was calculated by using the $2^{-\Delta \Delta C T}$ equation methods [42]. The PCR primers used in our study were shown in Supplementary Table 3. At least triple experiments were subjected to qRT-PCR verification.

\section{The expression levels of representative IncRNAs were verified in TANRIC database}

The Cancer Genome Atlas (TCGA, https://tcgadata.nci.nih.gov/tcga/) is a public funded project, which produces data at the RNA level for various cancers 
including lung adenocarcinom. The Atlas of Noncoding RNAs in Cancer (TANRIC, http://ibl.mdanderson.org/ tanric/_design/basic/index.html) is an open resource for interactive exploration of lncRNAs in the context of TCGA clinical and genomic data. In our study, TANRIC database was employed to retrieve the lncRNA expression profiling of LUAD from TCGA database. The difference of expression levels of representative lncRNAs between LUAD and adjacent non-tumor tissues were calculated, which were delineated by box-plot analysis.

\section{Receiver operating characteristic analyses}

In order to assess the diagnostic value of candidate DELs in LUAD, receiver operating characteristic (ROC) analyses were performed using $\mathrm{pROC}$ package in $\mathrm{R}$ language. The area under the curve (AUC) under binomial exact confidence interval was calculated to generate the ROC curve.

\section{Statistical analysis}

Mean \pm standard deviation and independent-samples $t$-test was used in the statistical analysis. $P<0.05$ was considered as significant difference. $*$ indicated $P<0.05$; $* *$ indicated $P<0.01$ and $* * *$ indicated $P<0.001$.

\section{Abbreviations}

LUAD: lung adenocarcinoma; lncRNA: long non-coding RNA; DEL: differentially expressed lncRNA; DEM: differentially expressed mRNA; DEMI: differentially expressed microRNA; KEGG: Kyoto Encyclopedia of Genes and Genomes; NSCLC: non-small cell lung cancer; EGFR-TKI: epidermal growth factor receptor tyrosine kinase inhibitor; ESCC: esophageal squamous cell carcinoma; GO: gene ontology; qRT-PCR: quantitative real-time polymerase chain reaction; ROC: receiver operating characteristic; AUC: area under the curve; PCC: Pearson correlation coefficient; TCGA: The Cancer Genome Atlas; TANRIC: The Atlas of Noncoding RNAs in Cancer.

\section{Author contributions}

Ziqiang Tian and Guiying Wang designed the experiments; Xinqiang Shi, Shiwang Wen and Yuefeng Zhang collected the patients' information; Xinqiang Shi, Yonggang Zhu, Yanzhao Xu and Huilai Lv searched the literatures; Ziqiang Tian, Shiwang Wen and Yuefeng Zhang analyzed the data; Guiying Wang and Ziqiang Tian wrote the manuscript and all authors reviewed the manuscript.

\section{ACKNOWLEDGMENTS}

\section{CONFLICTS OF INTEREST}

All of authors declare that they have none of conflicts of interest.

\section{FUNDING}

This study is supported by Special Project for the Transformation of Major Scientific and Technological Achievements of Hebei Province (No. 15277732D), Science and technology planning project of Hebei Province in 2015 (No. 15277732D), and Outstanding clinical Talent Training Project of Hebei Province in 2014.

\section{REFERENCES}

1. Siegel R, Naishadham D, Jemal A. Cancer statistics, 2012. CA Cancer J Clin. 2012; 62: 10-29. doi: 10.3322/ caac. 20138 .

2. Aokage K, Yoshida J, Hishida T, Tsuboi M, Saji H, Okada M, Suzuki K, Watanabe S, Asamura H. Limited resection for early-stage non-small cell lung cancer as functionpreserving radical surgery: a review. Jpn J Clin Oncol. 2016; 47: 7-11. doi: 10.1093/jjco/hyw148.

3. Provencio M, Isla D, Sanchez A, Cantos B. Inoperable stage III non-small cell lung cancer: current treatment and role of vinorelbine. J Thorac Dis. 2011; 3: 197-204. doi: 10.3978/j. issn.2072-1439.2011.01.02.

4. Kohler J, Schuler M. Afatinib, erlotinib and gefitinib in the first-line therapy of EGFR mutation-positive lung adenocarcinoma: a review. Onkologie. 2013; 36: 510-8. doi: $10.1159 / 000354627$.

5. Wei MM, Zhou GB. Long non-coding RNAs and their roles in non-small-cell lung cancer. Genomics Proteomics Bioinformatics. 2016; 14: 280-8. doi: 10.1016/j.gpb 2016.03.007.

6. Luo HL, Huang MD, Guo JN. AFAP1-AS1 is upregulated and promotes esophageal squamous cell carcinoma cell proliferation and inhibits cell apoptosis. Cancer Med. 2016; 5: 2879-85. doi: 10.1002/cam4.848.

7. Huang NS, Chi YY, Xue JY, Liu MY, Huang S, Mo M, Zhou $\mathrm{SL}, \mathrm{Wu}$ J. Long non-coding RNA metastasis associated in lung adenocarcinoma transcript 1 (MALAT1) interacts with estrogen receptor and predicted poor survival in breast cancer. Oncotarget. 2016; 7: 37957-65. doi: 10.18632/ oncotarget.9364.

8. Wang SH, Zhang WJ, Wu XC, Zhang MD, Weng MZ, Zhou D, Wang JD, Quan ZW. Long non-coding RNA Malat1 promotes gallbladder cancer development by acting as a molecular sponge to regulate miR-206. Oncotarget. 2016; 7: 37857-67. doi: 10.18632/oncotarget.9347.

9. Guo L, Wang J, Yang P, Lu Q, Zhang T, Yang Y. MicroRNA-200 promotes lung cancer cell growth through

Not applicable. 
FOG2-independent AKT activation. IUBMB Life. 2015; 67: 720-5. doi: 10.1002/iub.1412.

10. Ungewiss C, Rizvi ZH, Roybal JD, Peng DH, Gold KA, Shin DH, Creighton CJ, Gibbons DL. The microRNA-200/ Zeb1 axis regulates ECM-dependent beta1-integrin/FAK signaling, cancer cell invasion and metastasis through CRKL. Sci Rep. 2016; 6: 18652. doi: 10.1038/srep18652.

11. Wu XC, Wang SH, Ou HH, Zhu B, Zhu Y, Zhang Q, Yang Y, Li H. The NmrA-like family domain containing 1 pseudogene Loc344887 is amplified in gallbladder cancer and promotes epithelial-mesenchymal transition. Chem Biol Drug Des. 2017 Feb 28. doi: 10.1111/cbdd.12967. [Epub ahead of print]. doi: 10.1111/cbdd.12967.

12. Tejero R, Navarro A, Campayo M, Vinolas N, Marrades RM, Cordeiro A, Ruiz-Martinez M, Santasusagna S, Molins L, Ramirez J, Monzo M. miR-141 and miR-200c as markers of overall survival in early stage non-small cell lung cancer adenocarcinoma. PLoS One. 2014; 9: e101899. doi: 10.1371/journal.pone.0101899.

13. Lang $\mathrm{Y}, \mathrm{Xu} \mathrm{S}, \mathrm{Ma} \mathrm{J}, \mathrm{Wu} \mathrm{J}$, Jin $\mathrm{S}$, Cao $\mathrm{S}, \mathrm{Yu} \mathrm{Y}$. MicroRNA-429 induces tumorigenesis of human non-small cell lung cancer cells and targets multiple tumor suppressor genes. Biochem Biophys Res Commun. 2014; 450: 154-9. doi: 10.1016/j.bbrc.2014.05.084.

14. Nadal E, Truini A, Nakata A, Lin J, Reddy RM, Chang AC, Ramnath N, Gotoh N, Beer DG, Chen G. A novel serum 4-microRNA signature for lung cancer detection. Sci Rep. 2015; 5: 12464. doi: 10.1038/srep12464.

15. Zhu W, He J, Chen D, Zhang B, Xu L, Ma H, Liu X, Zhang Y, Le H. Expression of miR-29c, miR-93, and miR-429 as potential biomarkers for detection of early stage non-small lung cancer. PLoS One. 2014; 9: e87780. doi: 10.1371/ journal.pone.0087780.

16. Lin L, Gu ZT, Chen WH, Cao KJ. Increased expression of the long non-coding RNA ANRIL promotes lung cancer cell metastasis and correlates with poor prognosis. Diagn Pathol. 2015; 10: 14. doi: 10.1186/s13000-015-0247-7.

17. Gong WJ, Yin JY, Li XP, Fang C, Xiao D, Zhang W, Zhou $\mathrm{HH}, \mathrm{Li} \mathrm{X}$, Liu ZQ. Association of well-characterized lung cancer lncRNA polymorphisms with lung cancer susceptibility and platinum-based chemotherapy response. Tumour Biol. 2016; 37: 8349-58. doi: 10.1007/ s13277-015-4497-5.

18. Hu X, Bao J, Wang Z, Zhang Z, Gu P, Tao F, Cui D, Jiang $\mathrm{W}$. The plasma lncRNA acting as fingerprint in non-smallcell lung cancer. Tumour Biol. 2016; 37: 3497-504. doi: 10.1007/s13277-015-4023-9.

19. Zhao Y, Li Y, Lu H, Chen J, Zhang Z, Zhu ZZ. Association of copy number loss of CDKN2B and PTCH1 with poor overall survival in patients with pulmonary squamous cell carcinoma. Clin Lung Cancer. 2011; 12: 328-34. doi: 10.1016/j.cllc.2011.02.007.

20. Schuster K, Venkateswaran N, Rabellino A, Girard L, Pena-Llopis S, Scaglioni PP. Nullifying the CDKN2AB locus promotes mutant K-ras lung tumorigenesis. Mol Cancer Res. 2014; 12: 912-23. doi: 10.1158/1541-7786. mcr-13-0620-t.

21. Tian ZQ, Li ZH, Wen SW, Zhang YF, Li Y, Cheng JG, Wang GY. Identification of commonly dysregulated genes in nonsmall-cell lung cancer by integrated analysis of microarray data and qRT-PCR validation. Lung. 2015; 193: 583-92. doi: 10.1007/s00408-015-9726-6.

22. Weng TY, Wang CY, Hung YH, Chen WC, Chen YL, Lai MD. Differential expression pattern of THBS1 and THBS2 in lung cancer: clinical outcome and a systematic-analysis of microarray databases. PLoS One. 2016; 11: e0161007. doi: 10.1371/journal.pone.0161007.

23. Shen L, Yang M, Lin Q, Zhang Z, Zhu B, Miao C. COL11A1 is overexpressed in recurrent non-small cell lung cancer and promotes cell proliferation, migration, invasion and drug resistance. Oncol Rep. 2016; 36: 877-85. doi: 10.3892/or.2016.4869.

24. Hsu CP, Shen GH, Ko JL. Matrix metalloproteinase-13 expression is associated with bone marrow microinvolvement and prognosis in non-small cell lung cancer. Lung Cancer. 2006; 52: 349-57. doi: 10.1016/j. lungcan.2006.01.011.

25. Li Q, Wu C, Song G, Zhang H, Shan B, Duan Y, Wang Y. Genome-wide analysis of long noncoding RNA expression profiles in human Xuanwei lung cancer. Clin Lab. 2015; 61: 1515-23. doi: doi: 10.7754/Clin.Lab.2015.150323.

26. Miao L, Huang Z, Zengli Z, Li H, Chen Q, Yao C, Cai $\mathrm{H}$, Xiao Y, Xia H, Wang Y. Loss of long noncoding RNA FOXF1-AS1 regulates epithelial-mesenchymal transition, stemness and metastasis of non-small cell lung cancer cells. Oncotarget. 2016; 7: 68339-49. doi: 10.18632/ oncotarget.11630.

27. Wei HJ, Nickoloff JA, Chen WH, Liu HY, Lo WC, Chang YT, Yang PC, Wu CW, Williams DF, Gelovani JG, Deng WP. FOXF1 mediates mesenchymal stem cell fusioninduced reprogramming of lung cancer cells. Oncotarget. 2014; 5: 9514-29. doi: 10.18632/oncotarget.2413.

28. Wang H, Hao B, Chen X, Zhao N, Cheng G, Jiang Y, Liu Y, Lin C, Tan W, Lu D, Wei Q, Jin L, Lin D, et al. Beta-2 adrenergic receptor gene (ADRB2) polymorphism and risk for lung adenocarcinoma: a case-control study in a Chinese population. Cancer Lett. 2006; 240: 297-305. doi: 10.1016/j.canlet.2005.09.018.

29. Brogger J, Steen VM, Eiken HG, Gulsvik A, Bakke P. Genetic association between COPD and polymorphisms in TNF, ADRB2 and EPHX1. Eur Respir J. 2006; 27: 682-8. doi: 10.1183/09031936.06.00057005.

30. Piipponen M, Nissinen L, Farshchian M, Riihila P, Kivisaari A, Kallajoki M, Peltonen J, Peltonen S, Kahari VM. Long noncoding RNA PICSAR promotes growth of cutaneous squamous cell carcinoma by regulating ERK1/2 activity. J Invest Dermatol. 2016; 136: 1701-10. doi: 10.1016/j. jid.2016.03.028. 
31. Lu M, Tian H, Cao YX, He X, Chen L, Song X, Ping P, Huang H, Sun F. Downregulation of miR-320a/383sponge-like long non-coding RNA NLC1-C (narcolepsy candidate-region 1 genes) is associated with male infertility and promotes testicular embryonal carcinoma cell proliferation. Cell Death Dis. 2015; 6: e1960. doi: 10.1038/ cddis.2015.267.

32. Valles I, Pajares MJ, Segura V, Guruceaga E, GomezRoman J, Blanco D, Tamura A, Montuenga LM, Pio R. Identification of novel deregulated RNA metabolism-related genes in non-small cell lung cancer. PLoS One. 2012; 7: e42086. doi: 10.1371/journal.pone.0042086.

33. Wang YY, Wu ZY, Wang GC, Liu K, Niu XB, Gu S, Meng JS. LINC00312 inhibits the migration and invasion of bladder cancer cells by targeting miR-197-3p. Tumour Biol. 2016; 37: 14553-63. doi: 10.1007/s13277-016-5303-8.

34. Zheng ZG, Xu H, Suo SS, Xu XL, Ni MW, Gu LH, Chen W, Wang LY, Zhao Y, Tian B, Hua YJ. The essential role of $\mathrm{H} 19$ contributing to cisplatin resistance by regulating glutathione metabolism in high-grade serous ovarian cancer. Sci Rep. 2016; 6: 26093. doi: 10.1038/srep26093.

35. Jin X, Liu X. Integrated analysis of DNA methylation and mRNA expression profiles data to identify key genes in lung adenocarcinoma. Biomed Res Int. 2016; 2016: 4369431. doi: 10.1155/2016/4369431.
36. Trapnell C, Pachter L, Salzberg SL. TopHat: discovering splice junctions with RNA-Seq. Bioinformatics. 2009; 25: 1105-11. doi: 10.1093/bioinformatics/btp120.

37. Friedlander MR, Mackowiak SD, Li N, Chen W, Rajewsky N. miRDeep2 accurately identifies known and hundreds of novel microRNA genes in seven animal clades. Nucleic Acids Res. 2012; 40: 37-52. doi: 10.1093/nar/gkr688.

38. Benesty J, Chen J, Huang Y, Cohen I. Pearson correlation coefficient. Noise reduction in speech processing, 2nd Edition. Berlin: Springer, Berlin, Heidelberg; 2009. pp. 230.

39. Shannon P, Markiel A, Ozier O, Baliga NS, Wang JT, Ramage D, Amin N, Schwikowski B, Ideker T. Cytoscape: a software environment for integrated models of biomolecular interaction networks. Genome Res. 2003; 13: 2498-504. doi: $10.1101 /$ gr.1239303.

40. Dweep H, Gretz N. miRWalk2.0: a comprehensive atlas of microRNA-target interactions. Nat Methods. 2015; 12: 697. doi: 10.1038/nmeth.3485.

41. Carmona-Saez P, Chagoyen M, Tirado F, Carazo JM, Pascual-Montano A. GENECODIS: a web-based tool for finding significant concurrent annotations in gene lists. Genome Biol. 2007; 8: R3. doi: 10.1186/gb-2007-8-1-r3.

42. Schmittgen TD, Livak KJ. Analyzing real-time PCR data by the comparative CT method. Nat Protoc. 2008; 3: 1101-8. doi:10.1038/nprot.2008.73. 